\title{
Papers
}

\section{Risk of suicide and related adverse outcomes after exposure to a suicide prevention programme in the US Air Force: cohort study}

\author{
Kerry L Knox, David A Litts, G Wayne Talcott, Jill Catalano Feig, Eric D Caine
}

\begin{abstract}
Objective To evaluate the impact of the US Air Force suicide prevention programme on risk of suicide and other outcomes that share underlying risk factors.

Design Cohort study with quasi-experimental design and analysis of cohorts before (1990-6) and after (1997-2002) the intervention.

Participants 5260292 US Air Force personnel (around 84\% were men).

Intervention A multilayered intervention targeted at reducing risk factors and enhancing factors considered protective. The intervention consisted of removing the stigma of seeking help for a mental health or psychosocial problem, enhancing understanding of mental health, and changing policies and social norms.

Main outcome measures Relative risk reductions (the prevented fraction) for suicide and other outcomes hypothesised to be sensitive to broadly based community prevention efforts, (family violence, accidental death, homicide). Additional outcomes not exclusively associated with suicide were included because of the comprehensiveness of the programme.

Results Implementation of the programme was associated with a sustained decline in the rate of suicide and other adverse outcomes. A 33\% relative risk reduction was observed for suicide after the intervention; reductions for other outcomes ranged from $18-54 \%$.

Conclusion A systemic intervention aimed at changing social norms about seeking help and incorporating training in suicide prevention has a considerable impact on promotion of mental health. The impact on adverse outcomes in addition to suicide strengthens the conclusion that the programme was responsible for these reductions in risk.
\end{abstract}

\section{Introduction}

Suicide is a major public health problem in many industrialised countries, but there is little evidence of the effectiveness of prevention programmes. ${ }^{1}$ The low base rate of suicide makes it difficult to implement randomised trials that have sufficient statistical power when suicide is used as the major outcome. Synthesis of results from 20 randomised controlled trials of the effectiveness of psychosocial and drug treatments that used repetition of self harm as the outcome did not overcome this lack of power. ${ }^{2}$

From 1990-4 suicide rates in the US Air Force increased significantly overall $(\mathrm{P}<0.01)$, particularly among AfricanAmerican and white men aged 24-35 years. Senior figures in the US Air Force became concerned that suicide represented the end of a long road of personal suffering in which multiple indicators of vulnerability pointed to the need for help. They reasoned that this extended period of distress also offered an opportunity for preventive intervention. From their perspective, a responsible suicide prevention programme had to deal with the entire range of afflictions experienced by individuals, families, and their communities.

While many individuals have risk factors, only a few will ever attempt suicide. However, many exhibit decreased functioning, contributing to lost workdays, reduced productivity, great personal suffering, and substantial family distress. The uniqueness of the continuing programme has been its emphasis on early prevention, by intervening at the first signs of dysfunction or distress before the risk of suicide is imminent, while at the same time enhancing the detection and treatment of those at increased danger of taking their own lives. Early population based intervention to prevent suicide has been relatively uncommon. This may be partly due to the pervasive stigma in many cultures surrounding psychosocial or mental health problems, which deters individuals from seeking help..$^{3-5}$ These effects are compounded by poor understanding of mental health, defined as "knowledge and beliefs about mental disorders which aid their recognition, management or prevention." ${ }^{, 6}$ Fundamental to the approach taken by the Air Force was the understanding that only through reducing stigma could its community save lives.

During 1995 there were limited prevention efforts in selected groups of the Air Force and the suicide rate remained unacceptably high. In 1996, the Air Force implemented a population based prevention programme, involving community agencies inside and outside the healthcare sector. Emphasis was placed on institutionalising community-wide training efforts to heighten awareness of a range of risk factors that confer vulnerability for various behavioural and physical adverse events or problems, foremost of which was suicide. In addition, on an ongoing basis, the entire community received education about policy changes regarding the availability of resources to those in need. Finally, the senior ranks in the Air Force strongly endorsed a radical change in social norms to decrease stigma around help-seeking behaviours for all members of the community, and subsequently worked to sustain these newly stated values. We report on the effectiveness of the first six years of this ongoing programme, using a quasiexperimental (before and after) cohort study design.

\section{Methods}

Study population

The study population consisted of a dynamic cohort of 5260292 active duty US Air Force personnel between 1990-2002. 


\begin{tabular}{|c|c|c|}
\hline Initiatives and mandated policy & Action & Tracking indicators \\
\hline $\begin{array}{l}\text { I Leadership involvement (AFI 44-154 Suicide and Violence } \\
\text { Awareness and Education and Training) }\end{array}$ & $\begin{array}{l}\text { Leader awareness education and training (squadron } \\
\text { commander courses) }\end{array}$ & $\begin{array}{l}\text { Messages from USAF Chief of Staff delivered every 3-6 months to } \\
\text { all installation commanders reminding them of importance of } \\
\text { suicide prevention and encouraging them to actively promote } \\
\text { protective factors, identify risk factors, and encourage personnel } \\
\text { not to fear seeking help }\end{array}$ \\
\hline $\begin{array}{l}\text { II Dealing with suicide through professional military } \\
\text { education (AFI 44-154 Suicide and Violence Awareness } \\
\text { and Education and Training) }\end{array}$ & $\begin{array}{l}\text { Incorporate suicide prevention into professional military } \\
\text { education curriculums through required training }\end{array}$ & $\begin{array}{l}\text { Tracking of training, assessment of skills and knowledge of basic } \\
\text { suicide and violence risk factors, intervention skills, and referral } \\
\text { procedures for people potentially at risk }\end{array}$ \\
\hline $\begin{array}{l}\text { III Guidelines for commanders: use of mental health services } \\
\text { AFPAM 44-160 The Air Force Suicide Prevention Program }\end{array}$ & $\begin{array}{l}\text { Improve referrals of active duty members for evaluation of } \\
\text { mental health through emphasising that commanders and } \\
\text { mental health professionals are partners in improving duty } \\
\text { performance }\end{array}$ & $\begin{array}{l}\text { Annual briefings to commanders included resources for referral to } \\
\text { mental health, substance abuse, family advocacy, or emergency } \\
\text { evaluation (as of 2003, resources accessible through AF website } \\
\text { for commanders) }\end{array}$ \\
\hline IV Community preventive services (AF Manual 168-695) & $\begin{array}{l}\text { Increase preventive functions performed by mental health } \\
\text { personnel }\end{array}$ & $\begin{array}{l}\text { Provide one full time equivalent member of staff for community } \\
\text { based preventive services at every mental health work centre }\end{array}$ \\
\hline $\begin{array}{l}\text { V Community education and training (AFI 44-154 Suicide } \\
\text { Prevention Education and Community Training) }\end{array}$ & $\begin{array}{l}\text { Required training at two levels for non-professionals in basic } \\
\text { suicide factors, intervention skills, and referral procedures } \\
\text { for people potentially at risk }\end{array}$ & $\begin{array}{l}\text { Non-supervisory "buddy care" training for all personnel and } \\
\text { leadership/supervisory training for unit gatekeepers }\end{array}$ \\
\hline VI Investigative interview policy (hands-off policy) & $\begin{array}{l}\text { Changes in policies to ensure individuals under investigation } \\
\text { for legal problems (risk for suicide) are assessed for suicide } \\
\text { potential }\end{array}$ & $\begin{array}{l}\text { AF Chief of Staff signed policy letter in 1996; no suicides have } \\
\text { resulted since due to agencies failing to comply }\end{array}$ \\
\hline $\begin{array}{l}\text { VII Critical incident stress management (CISM) (AFI 44-153 } \\
\text { Critical Incident Stress Management) }\end{array}$ & $\begin{array}{l}\text { Establishment of a multidisciplinary CISM team to respond } \\
\text { to traumatic events, including completed suicides }\end{array}$ & $\begin{array}{l}\text { All installations now have multi-disciplinary CISM teams composed } \\
\text { of mental health providers, medical providers, and chaplains. }\end{array}$ \\
\hline $\begin{array}{l}\text { VIII Integrated delivery system (IDS) for human services } \\
\text { prevention, chartered as a standing subcommittee of (AF } \\
\text { CAIB AFI 90-500 Community Action Information Boards) }\end{array}$ & $\begin{array}{l}\text { Establishment of seamless system of services across } \\
\text { multidisciplinary human services prevention activities which } \\
\text { functions to provide centralised information (I) and referral } \\
\text { (R) and collaborative marking of IDS I and R and preventive } \\
\text { services }\end{array}$ & $\begin{array}{l}\text { Increase protective factors and decrease behavioural risk factors } \\
\text { through eliminating duplication, overlap, and gaps in delivering } \\
\text { prevention services. Core membership includes but not limited to } \\
\text { family advocacy programme, family support, health } \\
\text { promotion/health and wellness centres, mental health clinics, child } \\
\text { and youth programmes, and chaplains }\end{array}$ \\
\hline $\begin{array}{l}\text { IX Limited patient privilege (AFI 44-109 Mental Health, } \\
\text { Confidentiality and Military Law) }\end{array}$ & $\begin{array}{l}\text { Established psychotherapist-patient privilege for individuals } \\
\text { at risk for suicide as means to promote help seeking } \\
\text { behaviour }\end{array}$ & $\begin{array}{l}\text { Confidentiality encourages help seeking behaviour; especially in } \\
\text { cases undergoing disciplinary action where information revealed to } \\
\text { mental health provider is not used in judicial action }\end{array}$ \\
\hline X Behavioural health survey & $\begin{array}{l}\text { Tool for assessing behavioural health aspects of unit } \\
\text { available to any commander }\end{array}$ & $\begin{array}{l}\text { In } 1999 \text { survey } 73 \% \text { of commanders reported suicide was top item } \\
\text { of interest to understand how to promote behavioural health } \\
\text { strengths and respond to needs of their units }\end{array}$ \\
\hline XI Suicide event surveillance system & Central surveillance database & Tracks psychological, social, and behavioural risk factors \\
\hline
\end{tabular}

\section{Intervention}

In response to the rise in the numbers of suicides in 1990-5 the vice chief of staff mandated that prevention of suicide become a service-wide priority. He commissioned a team representing 15 functional areas (community based social service providers, operational components of the occupational community (command and supervision, safety), healthcare delivery, prevention and health promotion, and justice) and experts from the Centers for Disease Control to lead a systematic study of the issue and recommend a prevention strategy. The team used a data driven prevention model to examine extant community data and identified stigma, social norms, and commonly held beliefs that together apparently discouraged people from seeking help. The team adopted a population oriented risk reduction approach ${ }^{7}$ that focused on reducing modifiable risk factors and enhancing factors considered protective. (Reducing the overall mean population risk is always an important challenge because those bearing the highest risk often do not seek help on their own, whether through the healthcare delivery system, community based social services, colleagues, friends, or family.) Eleven "initiatives" were developed that targeted strengthening social support, promoting development of effective coping skills, and changing policies and norms so as to encourage effective help seeking behaviours (table 1). These initiatives are discussed in detail in Air Force Pamphlet 44-160, The Air Force Suicide Prevention Program (http://snowtrooper.epublishing.af.mil/pubs_catalog_rdb_lib/af/44/afpam44-160/ afpam44-160.pdf).

\section{Analysis}

Conceptual framework for evaluation-We carried out trend analyses on data routinely collected for other purposes. These included anonymised data collected in mortality databases for death due to all causes as well as data on judicial actions and family violence. To examine the question of whether implementation of the programme was associated with improved outcomes, the 1990-6 population was designated as the "unexposed" cohort and the population in 1997-2002 was considered to be the "exposed" cohort. (Although the programme was begun in 1996, it did not attain full implementation until 1997. Therefore, conservatively, any effects in 1996 were attributed to the time period before the intervention.) We considered that relative risk reductions (or the prevented fraction) in suicide and other related outcomes were an indication of the programme's overall effectiveness. We also investigated whether declines in the suicide rate could be ascribed to changes in demographics from 1990-2002; whether there had been an increase in the proportion of cases reported as homicides or accidental or undetermined deaths; and whether there was a greater proportion of individuals incapacitated due to a mental disorder. Timing of mandated policies service-wide was used as a measure that changes in practice occurred. Data on metrics of training and education about suicide and violence, which was one performance related indicator, were examined between 1997-2002.

Trend analysis of rates of suicide and related outcomes-We used $\chi^{2}$ test for trend with the Mantel-Haenszel statistic to test whether the rates for the main outcomes in each $\mathrm{n}$ level (year) were increasing or decreasing in a linear fashion. We used two sided $P$ values to test the null hypothesis of no difference over the decade and calculated $95 \%$ confidence intervals for each yearly rate to assess the precision of the rates.

Relative risk of suicide and related outcomes-We calculated relative risks (RR) as the ratio of the outcome of interest in the group exposed to the intervention after it was fully implemented 


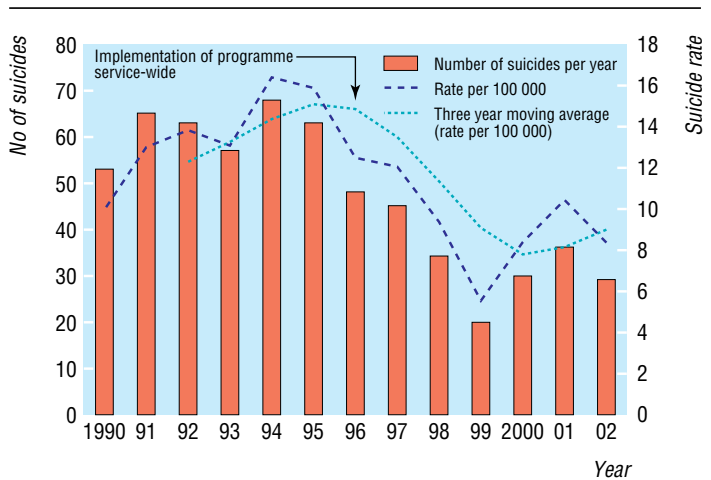

Fig 1 Number of suicides, suicide rates, and three year moving average for rates of suicide, US Air Force, 1990-2002

(1997-2002) to the outcome of interest in the group not exposed to the intervention (1990-6) calculated as:

$(\mathrm{RR})=\operatorname{Risk}_{\text {(exposed) }} / \operatorname{Risk}_{\text {(unexposed) }}=[\mathrm{a} /(\mathrm{a}+\mathrm{b})] /[\mathrm{c} / \mathrm{c}+\mathrm{d}]$

where $\mathrm{a}=$ subjects who were exposed to the intervention who had the outcome, $b=$ subjects exposed to the intervention who did not have the outcome, $\mathrm{c}=$ subjects not exposed to the intervention and who had the outcome, and $d=$ subjects not exposed to the intervention and who did not have the outcome. If exposure to the prevention programme had a protective effect (indicated by a relative risk under 1.0) the percentage of risk reduction (or preventive fraction) was calculated as $1-\mathrm{RR} \times 100$.

Population characteristics that might confound the analysesSuicide rates could be expected to decrease independent of the intervention if over the course of the study period there was an increased proportion of women, African-Americans, individuals younger than 25 or older than 34 , higher rank individuals (rank is considered a proxy for socioeconomic status), or married personnel or an increase in those discharged for a disability due to mental illness. We used a $\mathrm{z}$ test statistic and 95\% confidence intervals to determine whether there were significant differences in these population characteristics in the unexposed versus the exposed cohorts.

Changes in practice - Policy in the Air Force is governed by Air Force instructions and compliance with these instructions, at the installation level, is assessed regularly by the Air Force inspection agency. We conducted a historical document review to investigate the extent to which the 11 initiatives were mandated by a specific policy. We looked at training of all Air Force
Table 3 Comparison of effects of risk for suicide and related adverse outcomes in US Air Force population before (1990-6) and after implementation of programme (1997-2002)

\begin{tabular}{lccc} 
Outcome & $\begin{array}{c}\text { Relative risk } \\
\mathbf{( 9 5 \%} \mathbf{~ C l})\end{array}$ & $\begin{array}{c}\text { Risk reduction } \\
\text { (1-relative risk) }\end{array}$ & $\begin{array}{c}\text { Excess risk } \\
\text { (relative risk-1) }\end{array}$ \\
\hline Suicide & $0.67(0.57$ to 0.80$)$ & $33 \%$ & - \\
\hline Homicide & $0.48(0.33$ to 0.74$)$ & $51 \%$ & - \\
\hline Accidental death & $0.82(0.73$ to 0.93$)$ & $18 \%$ & - \\
\hline Severe family violence & $0.46(0.43$ to 0.51$)$ & $54 \%$ & - \\
\hline Moderate family violence & $0.70(0.69$ to 0.73$)$ & $30 \%$ & - \\
\hline Mild family violence & $1.18(1.16$ to 1.20$)$ & - & $18 \%$ \\
\hline
\end{tabular}

personnel in suicide awareness and prevention as an example of a performance objective.

\section{Results}

Trend analysis of rates of suicide and related outcomes-Analysis of the suicide data showed a linear trend for a reduction in the proportion of suicides each year across the decade $\left(\chi^{2}=11.3785\right.$, $\mathrm{df}=1, \mathrm{P}=0.0007)$. Analysis of the combined data on homicides and accidental deaths also indicated a linear trend for a decline in these outcomes $\left(\chi^{2}=5.3526, \mathrm{df}=1, \mathrm{P}=0.05\right)$. Analysis of the data on family violence found evidence for an increase in mild family violence $\left(\chi^{2}=1239.29, \mathrm{df}=1, \mathrm{P}<0.0001\right)$. There was a decrease in both moderate $\left(\chi^{2}=268.33, \mathrm{df}=1, \mathrm{P}<0.0001\right)$ and severe family violence $\left(\chi^{2}=227.14, \mathrm{df}=1, \mathrm{P}<0.0001\right)$. Figure 1 shows yearly rates and raw numbers for suicide from 1990-2002. Table 2 gives the estimated rates and the $95 \%$ confidence intervals for all of the outcomes for the years 1990-2002.

Relative risk of suicide and related outcomes-Table 3 gives details of relative risk and the preventive fraction for suicide and other violent outcomes. There was 33\% relative risk reduction of suicide in the exposed cohort; risk reductions for accidental death, homicide, and moderate and severe family violence ranged from $18-54 \%$. The relative risk of reported mild family violence was greater than 1 (relative risk $1.18,95 \%$ confidence interval 1.16 to 1.20 ), indicating there was an excess risk of the incidence of mild cases in the exposed cohort.

Analysis of potential population confounds-When we investigated potential demographic confounders we found no significant changes in sex, race, or age distribution in the cohort. There were also no differences in socioeconomic status (using rank as a proxy). The proportion of unmarried individuals rose slightly, though any confounding effect of this increase would serve to increase not decrease the risk of suicide. There was no

Table 2 Rates of suicide and other adverse outcomes (95\% confidence intervals) in US Air Force, 1990-2002

\begin{tabular}{|c|c|c|c|c|c|}
\hline \multirow[b]{2}{*}{ Year } & \multirow[b]{2}{*}{ Suicide per 100000} & \multirow{2}{*}{$\begin{array}{l}\text { Homicide/accidental death per } \\
100000\end{array}$} & \multicolumn{3}{|c|}{ Family violence per 1000} \\
\hline & & & Mild & Moderate & Severe \\
\hline 1990 & $10.0(7.3$ to 12.7$)$ & $20.7(16.9$ to 24.7$)$ & 4.1 (3.9 to 4.2$)$ & 2.7 (2.6 to 2.8$)$ & 0.37 (0.32 to 0.42$)$ \\
\hline 1991 & 13.0 (9.8 to 16.2$)$ & 28.9 (24.6 to 34.1$)$ & 8.9 (8.6 to 9.1$)$ & 5.3 (5.1 to 5.5$)$ & 0.86 (0.78 to 0.94$)$ \\
\hline 1992 & 13.8 (10.4 to 17.2$)$ & 23.4 (19.4 to 28.4$)$ & $9.9(9.6$ to 10.14$)$ & $5.9(5.6$ to 6.1$)$ & 0.98 (0.89 to 1.07$)$ \\
\hline 1993 & 13.1 (9.7 to 16.5$)$ & 25.9 (21.4 to 31.01$)$ & 11.1 (11.1 to 11.7$)$ & $6.4(6.1$ to 6.6$)$ & 0.96 (0.87 to 1.05$)$ \\
\hline 1994 & 16.4 (12.5 to 20.3$)$ & 23.7 (19.5 to 28.9 ) & 12.4 (12.1 to 12.7$)$ & 6.7 (6.4 to 6.9$)$ & 0.96 (0.87 to 1.05$)$ \\
\hline 1995 & $15.8(11.9$ to 19.7$)$ & 34.1 (28.2 to 39.6$)$ & 10.4 (10.1 to 10.7$)$ & $6.0(5.7$ to 6.2$)$ & 0.75 (0.66 to 0.82$)$ \\
\hline 1996 & 12.4 (8.9 to 15.9$)$ & 30.4 (24.8 to 35.8$)$ & $9.8(9.5$ to 10.1$)$ & 5.8 (5.6 to 6.1$)$ & 0.74 (0.65 to 0.82$)$ \\
\hline 1997 & $12.1(8.6$ to 15.6$)$ & 25.7 (19.6 to 20.6) & 7.6 (7.3 to 7.9$)$ & $4.0(3.8$ to 4.2$)$ & 0.45 (0.38 to 0.51$)$ \\
\hline 1998 & $9.4(6.3$ to 12.6$)$ & $22(17.2$ to 26.8$)$ & 10.2 (9.9 to 10.5$)$ & $5.3(5.0$ to 5.5$)$ & 0.46 (0.39 to 0.53$)$ \\
\hline 1999 & 5.6 (3.1 to 8.1$)$ & 16.6 (12.3 to 20.7) & $12.9(12.5$ to 13.3$)$ & $4.3(4.1$ to 4.5$)$ & 0.40 (0.34 to 0.47$)$ \\
\hline 2000 & 9.4 (6.2 to 12.7) & $14.0(10.1$ to 17.9$)$ & $11.9(11.5$ to 12.3$)$ & 3.3 (3.1 to 3.4$)$ & 0.26 (0.20 to 0.31$)$ \\
\hline 2001 & $10.4(7.0$ to 13.8$)$ & $21.3(16.5$ to 26.3$)$ & $12.2(11.8$ to 12.6$)$ & 3.0 (2.8 to 3.2$)$ & $0.28(0.22$ to 0.34$)$ \\
\hline 2002 & 8.3 (5.3 to 11.3$)$ & 25.3 (21.0 to 31.8$)$ & $11.7(11.3$ to 12.1$)$ & $3.2(3.1$ to 3.4$)$ & $0.38(0.32$ to 0.44$)$ \\
\hline
\end{tabular}




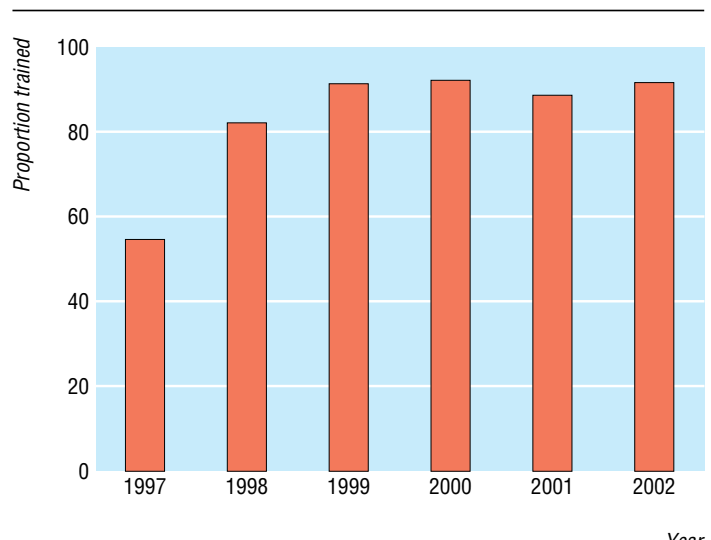

Fig 2 Proportion of Air Force personnel trained in suicide prevention (1997-2002)

difference in the two cohorts for the proportion of all disability cases that were assigned a mental health diagnosis, which would have caused them to be removed from active duty. Case reporting and overall distribution of deaths due to homicide, accidental death, undetermined causes, or suicide remained stable over the decade.

Changes in practice-The 11 initiatives were originally implemented through the release of Air Force instructions released early in 1997. All were associated with specific performance indicators (table 1). Figure 2 shows the proportion of people who received specific training in suicide and violence education; this increased significantly from 1997 to 2002 $\left(\chi^{2}=175677, \mathrm{df}=1, \mathrm{P}<0.0001\right)$.

\section{Discussion}

A community-wide suicide prevention programme aimed at decreasing stigma, enhancing social networks, facilitating help seeking through system level policy changes, and enhancing understanding of mental health in the community was associated over time with a 33\% risk reduction for completed suicide. Risk reductions of this magnitude observed in a clinical trial of a novel medication would attract immediate attention. ${ }^{8}$ We also observed significant risk reductions for accidental death, homicide, and moderate to severe family violence. "Moderate family violence" is defined in the US Air Force as exposure to repeated instances of emotionally abusive behaviour, neglect, or physical or sexual abuse; a report of "severe" abuse reflects a pattern of any of these that requires placement of the victim in an alternative environment. "Mild family violence" is defined as exposure to potentially harmful behaviour where there is not readily apparent physical or emotional harm. The Family Advocacy Program is one of the resources about which commanders were educated to enhance referral of individuals and families in distress. It has been a key component of the programme. The increase in the reported incidence of mild family violence may be related to an emphasis on outreach and earlier identification of distress. Indeed, although this may be a central component of the effectiveness of the prevention efforts, a causal association cannot be concluded from this study.

\section{Generalising the results}

As the US Air Force is a unique population for study, however, are the findings generalisable to other communities? While this is a concern, there is substantial value in examining such a setting. According to the theory of diffusion of innovations developed by Rogers and Shoemaker, ${ }^{9}$ individuals and communities who are first to implement novel interventions are designated as "early adopters." Though the Air Force community represents a select population, its development of community based suicide prevention is in keeping with its tradition of early implementation of public health innovations. ${ }^{10}$ Moreover, by studying this more stable population we were able to eliminate key confounders for risk of suicide, such as socioeconomic status and access to health care. While there is substantial diversity or heterogeneity within the US Air Force community, on the basis of educational level, financial resources, rank, job description, and installation assignment, the year to year variations in composition of the population as a whole are minimal, thus allowing the type of cohort analysis we used. This was confirmed by our examination of potential confounders. Scientific inference can be made more readily without confounding. Thus we consider that the ability to examine testable inferences about a theoretical model of suicide prevention was enhanced by studying this restricted population. ${ }^{11}$

Our findings support the theoretical models that form the foundation of the Air Force intervention. The fundamental goal of this population based risk reduction strategy was to decrease the mean population risk ${ }^{7}$ for a range of risk factors that confer vulnerability for various adverse behavioural and physical events or outcomes. That this shift occurred is unequivocal. Mandated changes in policy provide evidence that system level changes actually occurred. Measurements on training further support the conclusion that the Air Force fundamentally institutionalised suicide prevention training, which may have had far reaching mental health effects. Measuring changes in social norms is less straightforward. However, in a random survey conducted in 1999, 73\% of Air Force unit commanders identified risk of suicide as their highest concern regarding behavioural health in their units. Considering the low base rate of suicide and the relatively low probability of any one commander having a suicide in his or her unit, this level of concern is noteworthy in light of the social marketing campaign. Taken together with the sustained low rates of suicide and other adverse outcomes over the past six years, the social norm for help seeking behaviour (either for oneself or for a peer) seems to have shifted from being seen as a sign of weakness to a statement of strength and responsibility.

Community based approaches to health promotion present methodological challenges to study design and evaluation. The "noise" of real world environments often results in effect sizes smaller than expected. ${ }^{12}$ In contrast, we found reductions in risk similar to those seen after community interventions for HIV prevention ${ }^{13}$ that also have targeted changing social norms. ${ }^{14}$ The effect sizes measured in this cohort on several related indices of public health call for replication studies in other populations. The key lessons derived from this community based intervention may be particularly adaptable in selected workplace contexts that are more tightly organised and provide or coordinate human services for their employees or in settings with naturally occurring social networks. These could include police and fire fighters, other elements of the armed services worldwide, larger corporations, states or smaller countries, and schools and universities. The results also indicate that, for smaller preventive studies, examination of other theoretically related adverse outcomes can potentially yield important data regarding the prevention of suicide and the array of events that seem to be tied to it. 


\section{What is already known on this topic}

There is a lack of evidence for the effectiveness of broadly based community level suicide prevention programmes

The risk factors for suicide-including mental health problems, substance misuse, relationship problems, poor coping skills, legal and financial problems, and social isolation-also confer risk for other adverse outcomes, such as accidental death, homicide, and family violence

Little is known about the role that protective factors might have in preventing suicide, including increasing social support, coping skills, and understanding of mental health, and decreasing stigma about the related need to seek care

\section{What this study adds}

After the implementation of a broadly based, community level suicide prevention programme in the US Air Force, there were fewer suicides

The ongoing programme also reduced the rates of other adverse outcomes that share similar underlying risk factors

We thank John Nelson, Les Besetsny, Dari Tritt, Rick Campise, Kevin Grayson, James Whitworth, Katerina Neuhauser, and Len Wilson for providing data; Jane Pearson, Yeates Conwell, and Charles Hoge for their comments on the protocol; Paul Duberstein and Miriam Davis for providing critical feedback on the organisation of the final manuscript; and Charles Roadman II for pioneering a population approach to suicide prevention well before suicide became recognised as a public health problem.

Contributors: KLK, DAL, and EDC initiated the study and together with GWT and JCF designed the original protocol and coordinated the study KLK analysed the data. All the authors contributed to the revision of the protocol and the writing of the paper, which was initially drafted by KLK and DAL. KLK is guarantor for the study.

Funding: Department of Defense/Army/AMRDC, DAMD17-01-1-0797, R13 MH62073 (EDC), K24 MH01759 (Y Conwell), and K01 MH066317-01 (KLK).

Competing interests: None declared.
Ethical approval: Institutional Review Boards at the University of Rochester, the US Air Force, and the US Department of Defense.

1 Gunnell D, Frankel S. Prevention of suicide: aspirations and evidence. BMJ 1994 308:1227-33.

2 Hawton K, Arensman E, Townsend E, Bremner S, Feldman E, Goldney R et al. Deliberate self harm: systematic review of efficacy of psychosocial and pharmacological treatments in preventing repetition. BMJ 1998;317:441-7.

3 Link BG, Phelan JC, Bresnahan M, Stueve A, Pescosolido BA. Public conceptions of mental illness: labels, causes, dangerousness, and social distance. Am J Public Health 1999;89:1328-33.

4 Crisp AH, Gelder MG, Rix S, Meltzer HI, Rowlands OJ. Stigmatisation of people with mental illnesses. Br J Psychiatry 2000;177:4-7.

5 Link BG, Stuening EL, Rahav M, Phelan JC, Nuttbrock L. On stigma and its consequences: evidence from a longitudinal study of men with dual diagnoses of mental illness and substance abuse. J Health Soc Behav 1997;38:177-90.

6 Jorm AF. Mental health literacy. Public knowledge and beliefs about mental disorders Br J Psychiatry 2000;177:396-401.

7 Rose G. Sick individuals and sick populations. Int J Epidemiol 1985;14:32-8.

8 Sackett DL, Haynes RB, Guyatt GH, Tugwell P. Clinical epidemiology: a basic science for clinical medicine. Boston: Little, Brown,1991.

9 Rogers EM, Shoemaker EF. Communication of innovation. New York: Free Press, 1971:99-134.

10 Clements-Thompson M, Klesges RC, Haddock K, Lando H, Talcott W. Relationships between stages of change in cigarette smokers and healthy lifestyle behaviors in a population of young military personnel during forced smoking abstinence. J Counsult Clin Psychol 1998;66:1005-11.

11 Rothman KJ. Epidemiology. Oxford: University Press, 2002:110-1.

12 Merzel C, D'Affilitti J. Reconsidering community-based health promotion: promise, performance and potential. Am J Public Health 2003;93:557-74.

13 Kelly JA, St Lawrence JS, Stevenson LY, Hauth AC, Kalichman SC, Diaz YE, et al. Community AIDS/HIV risk reduction: the effects of endorsements by popular people in three cities. Am J Public Health 1992;82:1483-9.

14 Kelly JA. Community-level interventions are needed to prevent new HIV infections. Am J Public Health 1999;89:299-301.

(Accepted 10 October 2003)

bmj.com 2003;327:1376

University of Rochester Center for the Study and Prevention of Suicide, University of Rochester Medical Center, Rochester, NY 14642, USA

Kerry L Knox assistant professor of community and preventive medicine

Eric D Caine John Romano professor of psychiatry

Department of Health and Human Services, Washington, DC 20007, USA

David A Litts special adviser to US surgeon general for suicide prevention

Air Force Medical Operations Agency, Office of the Surgeon General, Brooks Air

Force Base, San Antonio, TX 78235, USA

$\mathrm{G}$ Wayne Talcott chief of community prevention division

Air Force Institute for the Environment, Safety and Occupation Health Risk

Analysis, Brooks Air Force Base, San Antonio

Jill Catalano Feig preventive medicine consultant

Correspondence to: K L Knox

Kerry_knox@urmc.rochester.edu 